\title{
Content based Image Retrieval System with Watermarks and Relevance Feedback
}

\author{
Sebin Jose \\ M-Tech Scholar \\ Computer Science and Systems Engineering \\ Govt.Engineering College,Idukki,Kerala,India
}

\author{
Philumon Joseph \\ Asst.Professor \\ Department of Computer Science and Engineering \\ Govt.Engineering College,Idukki,Kerala,India
}

\begin{abstract}
Image retrieval and related operations are always a 'hotspot' in the information era.Content-based image retrieval (CBIR) is a vastly developing area in the multimedia technology domain. To enhance security, we apply watermarking technique into the retrieval system and propose an approach for JEPG image retrieval. The proposed image retrieval system consists of three main phases, offline process, online retrieval process and the feedback process. The offline process aims at the feature vector extraction from the image. Later these features will be stored in the database. When it comes to the online retrieval process, it actually extracts the image features from the input image and matches these feature vectors with those available in the image database. In order to overcome the possible dissimilarity between bottom features and high-level semantics in the image retrieval; we introduce the feedback network to strengthen the retrieval efficiency.This is a simple categorized screening.Such a feedback scenario makes the system more user-friendly and effective.The proposed feedback screening strategy filters the images from irrelevant categories and enriches the final result with more relevant images.
\end{abstract}

\section{INTRODUCTION}

Content-based image retrieval (CBIR), as the name suggests is a fastest growing and interesting field in the multimedia world. We apply the watermarking technique into the retrieval system and propose a novel secure approach for JEPG image retrieval. Here, we have an existing system as a foundation to the proposed system.Color Image Retrieval System Based on Shape and Texture Watermarks : Hao Zhang, Hua Chen et. al [1] is the afforementioned system. The existing image retrieval method consists mainly of two phases, offline process and online retrieval process. In the offline process, the system will extract the features from each image.This extracted feature vector will be embedded into the image itself as a watermark.The second step is the online retrieval process. It consists of three sub processes, i.e., query feature computation where the features from the query image is extracted, watermark extraction from images and feature vector matching. Inorder to improve the accuracy of results we would like to enhance the system with a feedback screening methodology which actually chops off unwanted images from the final output. To be precise, we will have an image retrieval platform, which retrieves images based on the shapes and texture information. This system will be provided with a relevance feedback system which improves the image retrieval in later stages.

The image retrieval from web pages is cumbersome nowadays due to noise and lack of precise retrieval criterion. Such a method can enhance the image retrieval efficiency. Since it is provided with a relevance feedback system, its performance increases day by day for a particular user.The system will be extremely useful for a normal web user who randomly saves images during surfing. Normally images in websites are named as 'images.jpg' or 'index.jpg', hence the user will have to rename it while saving.Normally the users types some random names and save the images. So majority of the images will be having 'meaningless names'. So the Content based search will be more suitable here.

\section{LITERATURE SURVEY}

\subsection{Existing System}

The focus of study is Color Image Retrieval System Based on Shape and Texture Watermarks : Hao Zhang, Hua Chen et. al [1]. The various aspects regarding the existing system can be evaluated in detail.

2.1.1 Retrieval Platform Developed in Visual $C++$. This system has been implemented using Visual $\mathrm{C}++6.0$ software. It uses a standard image database including 1000 miscellaneous JPEG images of size $384 * 256$ or $256 * 384$, which are classified into ten classes, each class including 100 images. Euclidean distance is employed as the similarity measure.

The main attractions of this system are

- the system embeds the features in the images, and we need no extra space to save the feature data. Therefore, the storage space is saved in a way!

- There is no cross platform integration conflicts since it is purely developed in Visual C++

Apart from these advantages, it has certain major drawbacks.We must focus on the following drawbacks of this system. it is worthwhile to note that majority of the systems developed for the same objective faces these disadvantages as well.

- The system is developed in Visual $\mathrm{C}++$. So a user must execute a visual $\mathrm{C}++$ module to invoke the system. This is not convenient for a common user. 


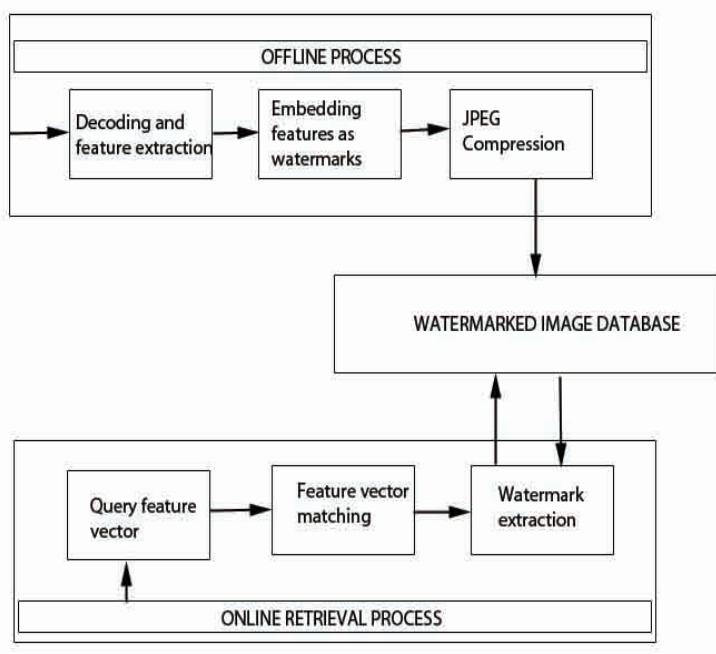

Fig. 1. The Existing image Retrieval system.

- It is not easy to integrate Visual $\mathrm{C}++$ modules with web interface. In the current scenario, majority of the users exploits web and web browsers as their most convenient medium of image retrieval. So they prefer a web based image retrieval platform.

-For a distributed system architecture such as 'Client-Server computing', it will be highly advantageous to employ a web based image retrieval platform. This is because, a system as mentioned above will demand each of the nodes to be installed with Visual $\mathrm{C}++$ modules and seperate databases. Whereas a web based system can allow the central server to be installed with the database and program, which can be executed by clients via their web browsers.

- The accuracy of results will be more important for certain applications rather than the retrieval speed. For example, the medical image retrieval platforms mainly focus on accuracy over speed. So a novel approach such as a feedback screening will be an added advantage to the image retrieval platform.

In order to bridge these disadvantages, Content Based Image Retrieval System with Watermarks and Relevance Feedback is proposed.

\section{PROPOSED METHOD}

The proposed system needs cross platform techniques to effectively achieve the goal.It is necessary to conduct a study on the various techniques available for the same. So the major concerns in the literature survey will be,

\section{-Feature extraction}

-Watermark Embedding

-Retrieval

—Feedback Mechanism

3.0.2 Feature extraction. Feature extraction is a form of dimensionality reduction. Which means that we reduce the dimensions of the actual object and grab necessary details only.This is used mainly when an algorithm is provided with a very large input and it is assumed to be notoriously redundant. For instance, the same measurement in both feet and meters. Then the best way is to convert the input data into a reduced representation set of features; and we call them as feature vectors. It is extremely important to ensure that the features are extracted carefully such that they will extract the relevant information from the input data. This is to perform the desired task using this reduced representation instead of the full size input without any reduction in accuracy. When performing analysis of sufficiently complex data, one of the usual overhead is the number of parameters involved. The processing with high number of parameters and variables generally requires a large amount of memory and computation power or a classification algorithm which overfits the training sample and generalizes poorly to new samples. The general dimensionality reduction techniques are listed below

—Principal component analysis

—Semidefinite embedding

-Multifactor dimensionality reduction

-Multilinear subspace learning

-Nonlinear dimensionality reduction

-Isomap

-Kernel PCA

-Multilinear PCA

—Latent semantic analysis

-Partial least squares

-Independent component analysis

-Autoencoder

It can be used in the area of image processing which involves using algorithms to detect and isolate various desired portions or shapes (features) of a digitized image or video stream. It is particularly important in the area of optical character recognition

Now, we can concentrate on the individual features of an image such as,

(1) Color,texture

(2) Moment Invariants

(3) Edge Information

Color features can be extracted from the images using bulit-in as well as custom designed MATLAB functions. Texture is a difficult concept to represent. The identification of specific textures in an image is achieved primarily by modelling texture as a two-dimensional gray level variation. The relative brightness of pairs of pixels is computed such that degree of contrast, regularity, coarseness and directionality may be estimated .However, the problem is in identifying patterns of co-pixel variation and associating them with particular classes of textures such as silky, or rough. Invariant moments computed over image have properties useful for image recognition - most notably independency from translation, rotation and scaling of images. Carefully chosen set of moments (just a vector of float values ) can be used to match against known characters using standard techniques for cluster analysis. Hu moments are most popular and well known set of moments to use for image recognition. 


\subsection{Watermark Embedding}

Watermarks are of two types; 'visible' and 'hidden'. Visible watermarks are intended to notify the users that the image is 'CopyRighted'. Hidden watermarks are mainly used to store secret information in the images. We use Visible watermarks to enhance the image security. There are vast number of methods available for watermarking. Some of them are,

\section{-Capacity estimation technique for JPEG watermaking —Quantization Index Modulation}

Each of these methods has its own advantages and disadvantages. Since we mainly focus on JPEG images, capacity estimation watermarking scheme can be employed efficiently in our system. The main aim of watermarking in our system is to ensure the security of images. Here we embed a visible watermark in the image during our post-feedback process. So each and every image in the final result will be having visible watermarks. The users need to exploitly search every images in the database.So the image database is made available to all the users. Watermarked images are helpful in such a scenario since the chance of misusing the images is higher.

3.1.1 Retrieval. Image retrieval platform should be a web application. Web pages may be used as the interface. We can develop it using html,php,javascript . At the back end, we may use a database ,preferably MySql . During the offline process, a MATLAB module will be invoked from the main webpage. It will generate the image database, consisting of the extracted features of images.

3.1.2 Feedback Mechanism. Feed back mechanism should be extremely simple. There is a tendency for the normal users to avoid the feedback after retrieving the results. So a scheme with simple radio buttons will be more handy than complex feedback mechanisms.Feedback mechanism targets to refine the results. The intermediete results will be presented to the user, he will select the irrelevant image(s); so the irrelevant categories will be removed from the final result.

\subsection{Proposed Method}

The proposed image retrieval system is designed based on the CBIR framework. The system consists of three main phases, offline process, online retrieval process and the feedback screening process. The offline process serves as a preprocessing operation for the image retrieval system. Its function is to form the watermarked image database, which contains the feature extraction and the data embedding. First, features such as color, texture, shape and spatial relationships are extracted from the input JPEG image. We test three kinds of feature vectors, seven moment invariants, the edge point histogram and the combined features (including color, shape and texture features).The extracted features are stored in the database. This is different from the existing system where the features are stored in individual images.

On the other hand, the online retrieval serves as the user interface. Since the features have been embedded into the database, it is not necessary to extract the features from images during the online retrieval. The strategies proposed for image retrieval systems basically approach content-based image retrieval algorithms by utilizing the similarity between the image in the database and the query image. Only those images, which have similar attributes as the query image, will be displayed to the user. In order to enhance the retreival accuracy, the feedback screening strategy is enriched within. The feedback form will prompt the user either to validate or invalidate the results. Based on which images from some particular

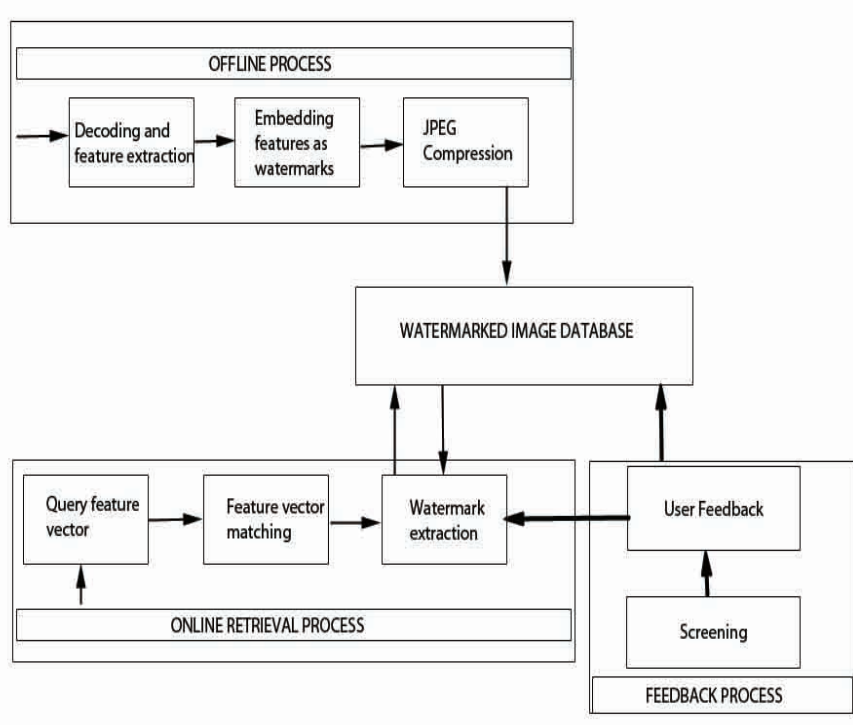

Fig. 2. The Proposed image Retrieval system.

category will be maintained, whereas invalid image categories will be screened off in the final results.

\subsection{Feature Computation}

In order to compute the features of the JPEG format image, we must decompress the image and obtain the original RGB pixels. Generally speaking, similarity between images is measured by computing the difference between their features such as color, shape, texture and spatial properties. This approach concentrates on three traditional image content descriptors, seven moment invariants, the edge histogram descriptor and the combination of color, shape, and texture descriptors. The feature vectors are all extracted based on the RGB color space. Shape features of objects or regions have been used in many content-based image retrieval systems. Classical shape representation used a set of moment invariants.

The edge histogram descriptor (EHD) describes the edge distribution of five types of edges in each area called a subimage. The sub-image is defined as an image block of size $128 * 128$. The edges in sub-images are categorized into five types, i.e., vertical, horizontal, 45-degree diagonal, 135-degree diagonal and non-directional edges. Thus, the histogram for each sub-image represents the relative frequency of occurrence of these 5 types of edges in the subimage. According to the template of each direction, edge detection is performed on each subimage and then the edge points of each sub-image are recorded.

The last kind of features presented are the combination of color, shape, and texture descriptors. The first two attributes are color invariants. The contrast (or inertial moment of the main diagonal), the energy and the entropy are used to describe the texture features.

\subsection{WATERMARK EMBEDDING AND DETECTION}

Watermarking in our system is only aimed at the security of images. It has nothing to do with the feature computation, feature extraction or feature embedding. The scenario was different in the previous system, where the image features are embedded into the image itself 
as a watermark.Modifying the system with a seperate database will help the user by avoiding the time and overhead needed to embedd and extract features from the image. So the term 'watermark' in the developing CBIR system stands only for the 'security purpose'.

\subsection{SCREENING BY FEEDBACK}

The most easiest method of 'direct feedbacks by the user' is adopted. Here, after the preliminary fetching the user will be provided with a screening page. This page essentially consists of radio buttons related to images. They can screen a particular image to be Appropriate, Inappropriate or No comments. Based on the user feedback, the inappropriate categories will be screened off. The final result will include more number of images from the appropriate category.

\section{IMPLEMENTATION}

The major part of the system is the Content Based Image Retrieval engine; this engine is the foundation for the entire system. CBIR engine is developed using MATLAB. We can categorize the implementation of the system into 3 major parts

(1) CBIR Engine developed in MATLAB

(2) Feedback network using Php+html

(3) Watermarking

\subsection{CBIR Engine developed in MATLAB}

MATLAB is the most powerful image processing tool available in the industry.It is possible to process images with great accuracy and precision by using MATLAB. MATLAB stands for Matrix Lab. Creation of database is the first step.There are 3 major categories of images. 'jpeg','png' and 'bmp'. Each of these images are read seperately.

For example

image=imread(fulfile(handles.flder_nme,jpg_files(jpg_count+1).nme)) Likewise, bmp and png files are also read.Now the images are resized to the prescribed resolution,

image $=$ imresize $($ image, $[384,256]$ );

After this , the main part of the dataset creation is carried out. The following three functions are used for dataset creation.

hsvHist=hsvHistogram(image)

autoCorrelogram=ColorAutoCorrelogram(image);

Color_Moments=ColorMoments(image);

The hsvHistogram function splits the image into $\mathrm{h}, \mathrm{s}$ and $\mathrm{v}$ planes. ColorAutoCorrelogram function measures and returns 4 predefined neighbour pixel intensities according to "image indexing using color correlogram[11]". The last function ColorMoments returns $1 * 6$ vector containing the 2 first color moments from each RGB channel. Also its mean and standard deviations are computed and stored in the dataset.

After getting all these results,they are saved onto the dataset variable set.

set $=[$ hsvHist autoCorrelogram Color_Moments meanAmplitude Wavelet_Moments];

That concludes the dataset creation part and the so called "Offline Process".

When it comes to the "Online Retrieval Process", the first step is selecting the image directory. Then the dataset is loaded, number of images to be returned is selected. After that we will select whether to use similarity metrics L1 or L2. Finally the image to be evaluated is selected and the database is queried.

\subsection{Feedback network using Php+html}

Once the preliminary On-line retrieval process gets completed, the user will be provided with a set of results based on his query. These images will be based purely on the algorithms L1 or L2, hence they may be from various domains. These images cannot be accessed by the users because of the security restrictions. Now, the feedback network will be invoked. It will prompt the user to select and thereby screen the invalid categories.

The php page will store the preliminary results in the database tables initially; then based on the feedback, the invalid entries will be removed from those tables. Finally the database will contain the end results only.

\subsection{Watermarking}

The main use of CBIR is correlated with 'Face database' . It can be effectively used in matching 'Face images'. Such a matching involves exclusive access over the face database images to any users.Obviously it is a security threat. So we introduces the watermarking methodology. The end results will not be published to the user directly. Instead, the user will be prompted to login with his credentials and the 'Watermarked images' will be returned to the user.

Watermarking is done using the simple php scripts which embeds mere text signatures on the images.

\section{CONCLUSIONS AND FUTURE WORK}

The image retrieval from web pages is cumbersome nowadays due to noise and lack of precise retrieval criterion.A content based method can enhance the image retrieval efficiency. Since it is provided with a relevance feedback system, its performance increases for a particular user based on his feedback choices. The system needs some extra space to store the image features. But it is not a disadvantage compared to the ease of extracting image features directly from the database;not from individual images. A content based image retrieval system is particularly useful to a common user who saves images randomly to a folder while surfing. Since there is very low probability for the user to name and categorize the image based on its content, it is too difficult to index them with names. Here we can use the CBIR approach.

There is vast scope for CBIR in many fields such as Security,Face recognition and pattern matching. The most obvious future work is working on facial database consisting of passport size images of peoples all around the globe. They will be categorized into various categories and the CBIR approach can effectively retrieve similar faces based on an input query image .

\section{ACKNOWLEDGMENT}

First and foremost, let me thank God almighty for the blessings over me to complete the project. Next I would like to thank my guide and co-author Asst.Prof Philumon Joseph for his continous encouragement. I would like to extend my heartful thanks to my parents and friends for their support. Last but not the least I sincerely thank all the authors of my reference papers, especially those who replied to my emails continously. Thank you

\section{DEVELOPED SYSTEM SCREEN SHOTS}




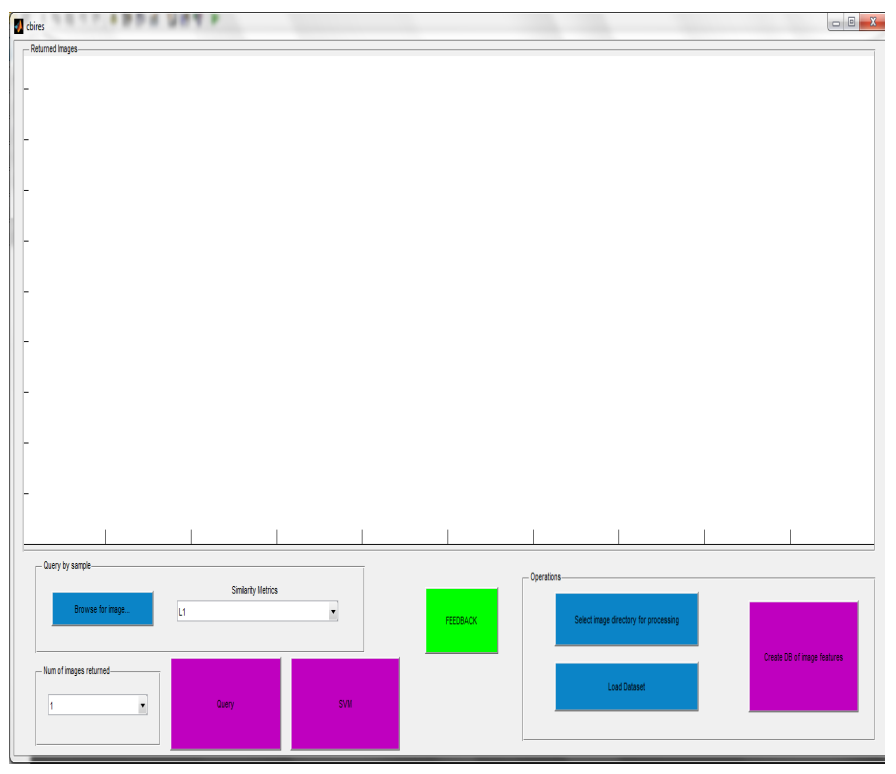

Fig. 3. CBIR System

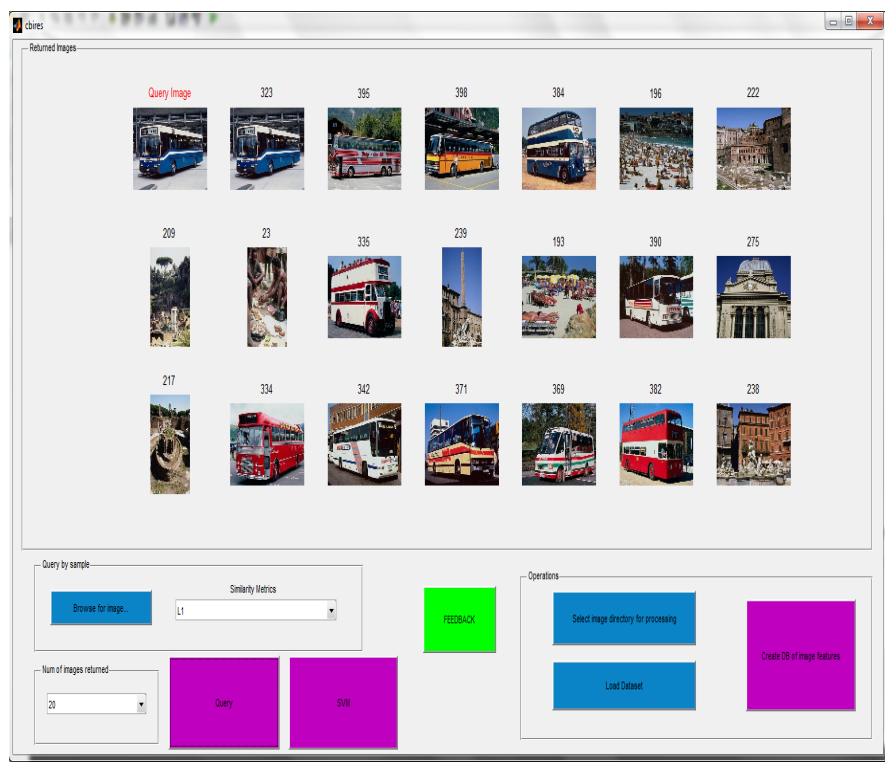

Fig. 4. Results after the Online process.

\section{REFERENCES}

[1] Color Image Retrieval System Based on Shape and Texture Watermarks : Hao Zhang, Hua Chen, Fa-Xin Yu and ZheMing Lu

[2] C. W. Niblack, R. Barber, W. Equitz, M. D. Flickner, E. H. Glasman, D. Pektovic, P. Yanker, C. Faloutsos, and G. Taubin, The QBIC Project: Querying Images by Content Using Color, Texture, and Shape," in: Proc. of Storage and Retrieval for Image and Video Databases, SPIE, vol. 1908, no.1, pp.173187, 1993.

[3] Image Retrieval Using ESNs and Relevance Feedback Yuan-

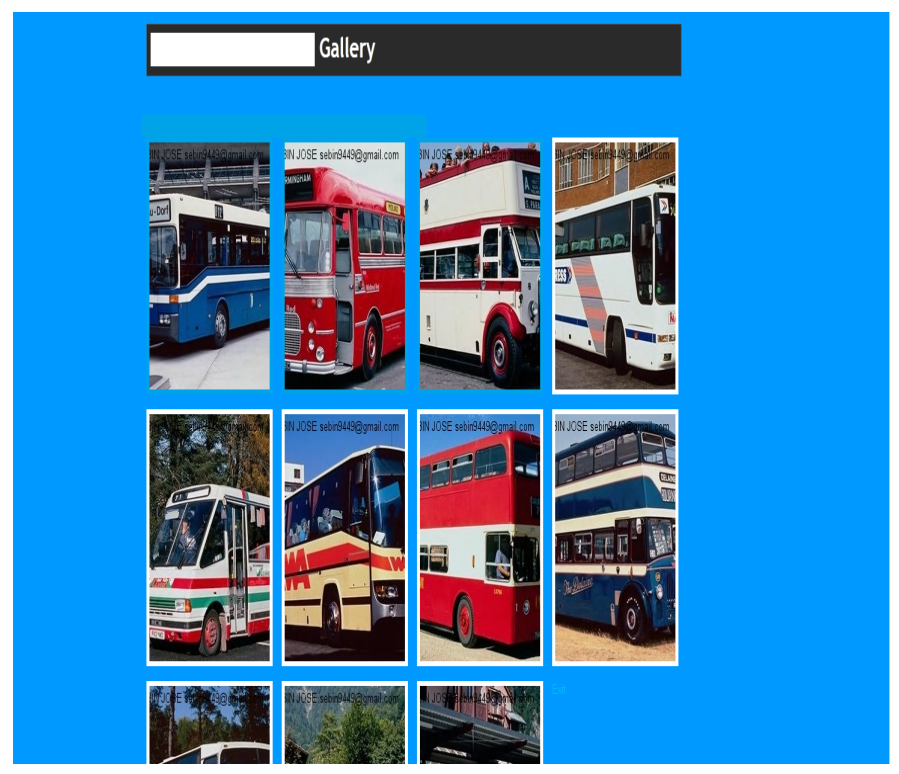

Fig. 5. Final Results.

feng Yang,JiangSu Province Support Software Engineering R\&D Center for Modern Information Technology Application in Enterprise Suzhou, China, 215104

[4] Information Hiding in Image Retrieval Systems,S. Areepongsa, N. Kaewkamnerd, Y. F. Syed, and K. R. Rao

[5] Quantization Index Modulation: A Class of Provably Good Methods for Digital Watermarking and Information Embedding , Brian Chen, Member, IEEE, and Gregory W. Wornell, Senior Member, IEEE

[6] A survey on content based image retrieval ,Dharani, T. ; Dept. of Comput. Sci., Periyar Univ., Salem, India ; Aroquiaraj, I.L. 2013 International Conference on Pattern Recognition, Informatics and Mobile Engineering (PRIME)

[7] Fusion of colour, shape and texture features for content based image retrieval,Anantharatnasamy, P. ; Dept. of Comput. Eng., Univ. of Peradeniya, Peradeniya, Sri Lanka ; Sriskandaraja, K. ; Nandakumar, V. ; Deegalla, S. 2013 8th International Conference on Computer Science \& Education (ICCSE)

[8] Content-Based Image Retrieval Using Invariant Color and Texture Features ,Afifi, A.J. ; Comput. Eng. Dept., Islamic Univ. of Gaza, Gaza, Palestinian Authority ; Ashour, W.M. 2012 International Conference on Digital Image Computing Techniques and Applications (DICTA)

[9] A novel public digital watermarking for still images based on encryption algorithm,Gwo-Chin Tai ; Dept. of Comput. Sci., Nat. Tsing Hua Univ., Hsinchu, Taiwan ; Long-Wen Chang IEEE 37th Annual 2003 International Carnahan Conference on Security Technology, 2003. Proceedings.

[10] A near reversible image watermarking algorithm,Bin Zhang ; Key Lab. of Network \& Inf. Attack \& Defence Technol. of MOE, Beijing Univ. of Posts \& Telecommun., Beijing, China ; Yang Xin ; Xin-Xin Niu ; Kai-Guo Yuan 2010 International Conference on Machine Learning and Cybernetics (ICMLC)

[11] A Similarity Measuring Method for Images Based on the Feature Extraction Algorithm using Reference Vectors, Ohno, A. ; 
Kobe Univ. Tsurukabuto, Kobe ; Murao, H. ICICIC '07. Second International Conference on Innovative Computing, Information and Control, 2007.

[12] Image indexing using color correlograms, Jing Huang Cornell Univ., Ithaca, NY, USA Kumar, S.R. ; Mitra, M. ; WeiJing Zhu ; Zabih, R.

[13] J. R. Smith and S. F. Chang, VisualSEEk: a fully automated content-based image query system, ACM Multimedia, Boston, MA., pp. 87-98, Nov. 1996.

[14] Y. Rui, T. S. Huang and S. Chang, Image Retrieval: current techniques, promising directions, and open issues, J. Visual Communication and Image Representation, vol. 10, pp. 3962, March 1999.

[15] S. Areepongsa and K.R. Rao, Invariant features for texture image retrieval using steerable pyramid, WPMC 2000, Bangkok, Thailand, Nov. 2000.

[16] H. Mller, W. Mller, D. Squire, S. Marchand-Maillet, and T. Pun, Performance Evaluation in Content-based Image Retrieval: Overview and Proposals, Pattern Recognition Letters, vol. 22, no. 5, pp. 593601, Apr. 2001.

[17] X. L. Li, Watermarking in Secure Image Retrieval, Patten Recognition Letters, vol. 24,no.14, pp. 24312434, Oct. 2003. 